\title{
Turbulent superfluid as continuous vortex mixture
}

\author{
V.I. Yukalov \\ Bogolubov Laboratory of Theoretical Physics, \\ Joint Institute for Nuclear Research, Dubna 141980, Russia \\ and \\ National Institute of Optics and Photonics, \\ University of São Paulo, São Carlos 13560-970, Brazil
}

\begin{abstract}
A statistical model is advanced for describing quantum turbulence in a superfluid system with Bose-Einstein condensate. Such a turbulent superfluid can be realized for trapped Bose atoms subject to either an alternating trapping potential or to an alternating magnetic field modulating the atomic scattering length by means of Feshbach resonance. The turbulent system is represented as a continuous mixture of states each of which is characterized by its own vorticity corresponding to a particular vortex.
\end{abstract}

Key words: Quantum turbulence; turbulent superfluid; statistical model of turbulence; oscillating trap modulation; trapped atoms

PACS: 67.10.Ba, 67.10.Fj, 67.25.dk, 67.85.De, 67.85.Jk, 47.27.eb, 47.27.Gs, 03.75.Kk, 03.75.Lm, 03.75.Nt, 05.30.Ch, 05.30.Jp, 05.45.Yv, 05.70.Ln 


\section{Quantum turbulence}

Turbulence in classical fluids has been intensively studied for many years [1-5]. It can also occur in superfluids, such as ${ }^{4} \mathrm{He},{ }^{3} \mathrm{He}$, and trapped atomic gases. A superfluid differs from a classical fluid in three principal respects: it exhibits two-fluid behavior; the superfluid component can flow without dissipation; and flow of the superfluid component is subject to quantum restrictions. One of the major such restrictions is that the vortices in superfluids are quantized [6-11]. Emphasizing that the peculiarities of turbulence in superfluids, as compared to that in classical fluids, are due to quantum effects, turbulence in superfluids is called quantum turbulence [12-14].

There are several ways of producing turbulence in superfluids, e.g., by realizing counterflows between the normal and superfluid components, by moving grids or by vibrating objects immersed in the superfluid [12-14]. The fully developed quantum turbulence is represented by a random complex tangle of quantized vortices. Their appearance can be due to different reasons, to mutual friction between the normal and superfluid components [12-16], accompanied by the Kelvin-Helmholtz instability $[17,18]$, to thermal and quantum tunneling [12], and to other dynamic instabilities $[19,20]$. When the external disturbance, producing turbulence, ceases to act on the fluid, turbulence decays through the Richardson cascades [12-14], the emission of sound radiation produced by vibrating vortices [21,22], by vortex reconnecion [23,24], and by Kelvin-wave cascades [25,26]. The evolution of quantized vortices can be visualized by injecting into the fluid small admixture particles [27].

Bose-Einstein condensed gases of trapped atoms [11, 28-33] provide additional possibilities for studying superfluids in a well controlled way. However, by rotating trapped Bose condensates, one can create quantized vortices, which tend to form crystalline structures, when the rotation velocity increases [34-36]. Simple rotation does not produce turbulence in trapped systems. In order to create a tangle of vortices, typical of quantum turbulence, it is necessary to use some other techniques. Actually, a vortex is a particular case of coherent topological modes, among which there exist many other soliton-like excitations [37,38]. Hence, the general question is how it would be possible to create coherent topological modes for trapped atomic systems?

A method for generating various coherent topological modes in traps has been advanced, based on the modulation of atomic cloud density [39-41]. The modulation can be done in two ways, either by oscillating trapping potentials [39-43] or by alternating the atomic scattering length using Feshbach resonance techniques [33,44,45]]. Both these ways yield similar results, so that it is a matter of convenience which of them to employ. The generation of the coherent modes can be achieved by rather weak modulation amplitudes, but provided that the frequency of the alternating trapping potential or of the alternating scattering length is in resonance with the transition frequency corresponding to the desired coherent mode [3945]. By increasing the modulation amplitude, it is feasible to realize the nonresonant mode generation [42]. In particular, the generation, without the direct resonance condition, can be done, e.g., by means of parametric conversion or harmonic generation [46,47]. Very strong forced oscillations can even destroy superfluidity at all [48]. The alternating modulation of trapping potentials, to some extent, can be modelled by combined rotations around several axes [49].

For theoretical description of quantum turbulence, it is sufficient to consider the nonlinear Schrödinger equation (Gross-Pitaevskii equation) [49,50], keeping in mind low temperatures 
and weak atomic interactions, when almost the whole system is in the Bose-condensed state. The generation of coherent topological modes and, hence, turbulence, can also be realized under more general conditions of finite temperatures and strong atomic interactions [51]. The presence of the normal component is not necessary for the appearance of quantized vortices. The latter arise because of the occurrence of a heteroclinic instability [38] in a nonequilibrium system, when the homogeneous atomic density becomes dynamically unstable with respect to its transformation to a nonuniform density comprising topological defects, like quantized vortices. This is analogous to the development of classical turbulence that is accompanied by a strongly nonuniform distribution of density [52-55]. In quantum turbulence, the nucleation of vortices, under the dynamic instability, is caused by developing unstable collective excitations, such, e.g., as quadrupole modes $[19,20]$. The typical feature of quantum turbulence is the random spatial distribution of quantized vortices [12-14], similar to the models of dynamical glass [56].

Experimentally, the modulation of the trapping potential has been used for the generation of quantized vortices [57] and vortex tangles [58]. The appearance of vortex tangles implies that the system enters the fully turbulent regime [12-14].

Turbulence is a strongly nonequilibrium phenomenon representing an essentially nonuniform matter. This is why it is difficult for theoretical description. But, in the case of fully developed stationary turbulence, it can be possible to invoke statistical methods. It is the aim of the present paper to suggest a statistical model of stationary quantum turbulence.

\section{Vortex mixture}

Quantized vortices are the necessary ingredients of quantum turbulence. Each vortex can be represented as a filament passing through the fluid. The velocity $\mathbf{v}=\mathbf{v}(\mathbf{r})$ at a point $\mathbf{r}$, due to a filament, is given by the Biot-Savart formula

$$
\mathbf{v}=\frac{n}{2 m} \int \frac{(\mathbf{s}-\mathbf{r})}{|\mathbf{s}-\mathbf{r}|^{3}} \times d \mathbf{s},
$$

where $m$ is atomic mass, $n$ is an integer characterizing the quantum of circulation, the vector $\mathbf{s}$ refers to a point on the filament, and the integration is taken along the filament. The filament has a definite direction associated with its vorticity

$$
\vec{\omega} \equiv \nabla \times \mathbf{v} .
$$

Integrating velocity along a path around the vortex core gives the quantum of circulation,

$$
\oint \mathbf{v} \cdot d \mathbf{l}=\frac{2 \pi n}{m} \quad(n=0, \pm 1, \pm 2, \ldots) .
$$

For example, in the case of a rectilinear vortex, with the vorticity along the axis $z$, the velocity is

$$
\mathbf{v}=\frac{n}{m r_{\perp}} \mathbf{e}_{\varphi}=\frac{n}{m r_{\perp}^{2}} \mathbf{e}_{z} \times \mathbf{r}_{\perp},
$$

where $r_{\perp} \equiv \sqrt{x^{2}+y^{2}}=\left|\mathbf{r}_{\perp}\right|$. This gives the vorticity

$$
\vec{\omega}_{n}=\frac{2 \pi n}{m} \delta(x) \delta(y) \mathbf{e}_{z} .
$$


The case of $n=0$ means the absence of a vortex.

In the turbulent superfluid there are many vortices with different vorticities, whose distribution can be characterized by a measure $m(\vec{\omega})$. Thus, in the case of rectilinear vortices, integration of a function $f(\vec{\omega})$, with a measure $m(\vec{\omega})$, gives

$$
\int f(\vec{\omega}) d m(\vec{\omega})=\sum_{n} \int f\left(\vec{\omega}_{n}\right) d \Omega\left(\mathbf{e}_{z}\right)
$$

where summation is over the circulation quanta $n=0,1,2, \ldots$ and integration is over the spherical angle around $e_{z}$, that is, over all directions of vorticity.

A fluid with a quantized vortex represents a dynamic state that is principally different from the state without vortices. Moreover, the states with the vortices, having different winding numbers or vorticities can be treated as different states. At any instant of time, the system, formed by multiple regions, each containing a vortex, can be considered as a composition of states corresponding to these different regions. So, at each instant of time, the system is represented as a configuration, composed of regions with different vortices. The density of vortices in the system and their directions are random in space and are varying in time. This means that the vortex configuration varies. The statistical description of such a situation assumes an averaged picture corresponding to the averaging over all admissible state configurations. The theory of describing such heterophase mixtures has been advanced in [59-63] and reviewed in [64-66], where all mathematical details of the related averaging procedure are elucidated.

Following this approach [64-66], we come to an effective Hamiltonian characterizing the random mixture. In the present case, this Hamiltonian is the direct integral

$$
\widetilde{H}=\int^{\oplus} H(\vec{\omega}) d m(\vec{\omega}) \bigoplus H_{2}
$$

Here $H(\vec{\omega})$ is the Hamiltonian of a system with a vortex characterized by the vorticity $\vec{\omega}$ and $H_{2}$ is the Hamiltonian corresponding to the normal (nonsuperfluid) state. The latter has to be taken into account since, in the process of generating turbulence, superfluidity can be destroyed in a part of the system. This Hamiltonian (4) is defined on the mixture space

$$
\mathcal{M}=\bigotimes_{\vec{\omega}} \mathcal{H}(\vec{\omega}) \bigotimes \mathcal{H}_{2}
$$

which is a continuous tensor product of the weighted Hilbert spaces. More details on the meaning of continuous products are given in the Appendix. Each weighted space $\mathcal{H}(\vec{\omega})$ is a copy of the system Hilbert space, with a weighted scalar product [64-66], corresponding to the state with a vortex labelled by its vorticity $\vec{\omega}$. And $\mathcal{H}_{2}$ is a space corresponding to the normal (nonsuperfluid) state.

The superfluid states with the vortices are described by the grand Hamiltonians

$$
H(\vec{\omega})=\hat{H}(\vec{\omega})-\mu_{0}(\vec{\omega}) N_{0}(\vec{\omega})-\mu_{1}(\vec{\omega}) \hat{N}_{1}(\vec{\omega})
$$

in which $\hat{H}(\vec{\omega})$ is the energy operator, $N_{0}(\vec{\omega})$ is the number of condensed atoms, $\hat{N}_{1}(\vec{\omega})$ is the number-of-particle operator for uncondensed atoms, and $\mu_{0}(\vec{\omega})$ and $\mu_{1}(\vec{\omega})$ are the Lagrange multipliers guaranteeing the normalization conditions for the number of Bose-condensed 
atoms $N_{0}(\vec{\omega})$ and for the number of uncondensed atoms $N_{1}(\vec{\omega})$ in the superfluid state of vorticity $\vec{\omega}[51,67-69]$. The nonsuperfluid state is characterized by the grand Hamiltonian

$$
H_{2}=\hat{H}_{2}-\mu_{2} \hat{N}_{2}
$$

where $\hat{H}_{2}$ is the corresponding energy operator, $\hat{N}_{2}$ is the related number-of-particle operator, and $\mu_{2}$ is the chemical potential of the normal state.

In what follows, we shall consider local atomic interactions

$$
\Phi(\mathbf{r})=\Phi_{0} \delta(\mathbf{r}), \quad \Phi_{0} \equiv 4 \pi \frac{a_{s}}{m},
$$

where $m$ is atomic mass and $a_{s}$, scattering length. The local form (8) is not principal, but is taken just for the brevity of notations. Throughout the paper, the units are employed, where $\hbar=1$ and $k_{B}=1$.

The Hamiltonian energy operator in (6) has the form

$$
\begin{gathered}
\hat{H}(\vec{\omega})=w_{1}(\vec{\omega}) \int \hat{\psi}^{\dagger}(\vec{\omega}, \mathbf{r})\left[\frac{\hat{\mathbf{p}}^{2}}{2 m}+U(\mathbf{r})\right] \hat{\psi}(\vec{\omega}, \mathbf{r}) d \mathbf{r}+ \\
+\frac{w_{1}^{2}(\vec{\omega})}{2} \Phi_{0} \int \hat{\psi}^{\dagger}(\vec{\omega}, \mathbf{r}) \hat{\psi}^{\dagger}(\vec{\omega}, \mathbf{r}) \hat{\psi}(\vec{\omega}, \mathbf{r}) \hat{\psi}(\vec{\omega}, \mathbf{r}) d \mathbf{r}
\end{gathered}
$$

where the field operators $\hat{\psi}(\vec{\omega}, \mathbf{r})$ act on the space $\mathcal{H}(\vec{\omega})$ and the operator of momentum is $\hat{\mathbf{p}} \equiv-i \nabla$. The factor $w_{1}(\vec{\omega})$ is the geometric weight of the superfluid vortex state with vorticity $\vec{\omega}$. The function $U(\mathbf{r})$ is an external potential, say, a trapping potential. The superfluid system is assumed to be Bose-condensed. And the Bose-Einstein condensation necessarily requires that the global gauge symmetry be broken [70].

The most convenient way of gauge symmetry breaking is by means of the Bogolubov shift [71], which can be represented as the sum

$$
\hat{\psi}(\vec{\omega}, \mathbf{r})=\eta(\vec{\omega}, \mathbf{r}) \exp \{i S(\mathbf{r})\}+\psi_{1}(\vec{\omega}, \mathbf{r})
$$

in which $S(\mathbf{r})$ is a real function, such that

$$
\mathbf{v}=\mathbf{v}(\mathbf{r})=\frac{1}{m} \nabla S(\mathbf{r})
$$

is the superfluid velocity. The first term in (10) is the condensate wave function and the second term is an operator of uncondensed atoms in the superfluid state with vorticity $\vec{\omega}$. To exclude the double counting, the terms in sum (10) are to be orthogonal to each other,

$$
\int \eta(\vec{\omega}, \mathbf{r}) \exp \{i S(\mathbf{r})\} \hat{\psi}_{1}^{\dagger}(\vec{\omega}, \mathbf{r}) d \mathbf{r}=0
$$

And the statistical average for the operator of uncondensed atoms satisfies the property

$$
\left\langle\psi_{1}(\vec{\omega}, \mathbf{r})\right\rangle=0
$$

due to which the condensate wave function plays the role of the order parameter. 
The Hamiltonian energy operator in (7) is

$$
\begin{aligned}
\hat{H}_{2} & =w_{2} \int \psi_{2}^{\dagger}(\mathbf{r})\left[\frac{\hat{\mathbf{p}}^{2}}{2 m}+U(\mathbf{r})\right] \psi_{2}(\mathbf{r}) d \mathbf{r}+ \\
& +\frac{w_{2}^{2}}{2} \Phi_{0} \int \psi_{2}^{\dagger}(\mathbf{r}) \psi_{2}^{\dagger}(\mathbf{r}) \psi_{2}(\mathbf{r}) \psi_{2}(\mathbf{r}) d \mathbf{r}
\end{aligned}
$$

where the field operators $\psi_{2}(\mathbf{r})$, acting on $\mathcal{H}_{2}$, correspond to the normal (nonsuperfluid) state and $w_{2}$ is the geometric weight of the normal state. All field operators satisfy the Bose commutation relations.

The condensate wave function defines the density of condensed atoms in the state with vorticity $\vec{\omega}$,

$$
\rho_{0}(\vec{\omega}, \mathbf{r})=|\eta(\vec{\omega}, \mathbf{r})|^{2} .
$$

The density of uncondensed atoms in the superfluid state, labelled by $\vec{\omega}$, is

$$
\rho_{1}(\vec{\omega}, \mathbf{r})=\left\langle\psi_{1}^{\dagger}(\vec{\omega}, \mathbf{r}) \psi_{1}(\vec{\omega}, \mathbf{r})\right\rangle .
$$

And the density of the normal phase is

$$
\rho_{2}(\mathbf{r})=\left\langle\psi_{2}^{\dagger}(\mathbf{r}) \psi_{2}(\mathbf{r})\right\rangle .
$$

The number of condensed atoms in the $\vec{\omega}$ vortex state is

$$
N_{0}(\vec{\omega})=w_{1}(\vec{\omega}) \int \rho_{0}(\vec{\omega}, \mathbf{r}) d \mathbf{r} .
$$

The number-of-particle operator of uncondensed atoms in that state is

$$
\hat{N}_{1}(\vec{\omega})=w_{1}(\vec{\omega}) \int \psi_{1}^{\dagger}(\vec{\omega}, \mathbf{r}) \psi_{1}(\vec{\omega}, \mathbf{r}) d \mathbf{r} .
$$

And the number-of-particle operator for atoms in the normal state is

$$
\hat{N}_{2}=w_{2} \int \psi_{2}^{\dagger}(\mathbf{r}) \psi_{2}(\mathbf{r}) d \mathbf{r} .
$$

So that the number of uncondensed atoms in the $\vec{\omega}$ vortex state reads as

$$
N_{1}(\vec{\omega}) \equiv\left\langle\hat{N}_{1}(\vec{\omega})\right\rangle=w_{1}(\vec{\omega}) \int \rho_{1}(\vec{\omega}, \mathbf{r}) d \mathbf{r},
$$

and the number of normal atoms being

$$
N_{2} \equiv\left\langle\hat{N}_{2}\right\rangle=w_{2} \int \rho_{2}(\mathbf{r}) d \mathbf{r} .
$$

The number of condensed atoms in all vortex states is the integral

$$
N_{0}=\int N_{0}(\vec{\omega}) d m(\vec{\omega}),
$$


while the number of uncondensed atoms in all vortex states is

$$
N_{1}=\int N_{1}(\vec{\omega}) d m(\vec{\omega}) .
$$

The total number of atoms in the system is

$$
N=N_{0}+N_{1}+N_{2}
$$

The geometric weight of the superfluid state with all types of vortices reads as

$$
w_{1} \equiv \int w_{1}(\vec{\omega}) d m(\vec{\omega})
$$

There can be in the system only two types of states, superfluid and normal, which implies the normalization

$$
w_{1}+w_{2}=1 \quad\left(0 \leq w_{\nu} \leq 1\right)
$$

where $\nu=1,2$.

The equations of motion for the field variables are given by the related variations: for the condensate wave function of a superfluid state with the vorticity $\vec{\omega}$,

$$
i \frac{\partial}{\partial t} \eta(\vec{\omega}, \mathbf{r}, t)=\left\langle\frac{\delta \widetilde{H}}{\delta \eta^{*}(\vec{\omega}, \mathbf{r}, t)}\right\rangle ;
$$

for the field operator of uncondensed atoms in the superfluid state with the vorticity $\vec{\omega}$,

$$
i \frac{\partial}{\partial t} \psi_{1}(\vec{\omega}, \mathbf{r}, t)=\frac{\delta \widetilde{H}}{\delta \psi_{1}^{\dagger}(\vec{\omega}, \mathbf{r}, t)}
$$

and for the field operator of atoms in the normal (nonsuperfluid) state,

$$
i \frac{\partial}{\partial t} \psi_{2}(\mathbf{r}, t)=\frac{\delta \tilde{H}}{\delta \psi_{2}^{\dagger}(\mathbf{r}, t)} .
$$

Let us introduce the notations for the anomalous average

$$
\sigma_{1}(\vec{\omega}, \mathbf{r}) \equiv\left\langle\psi_{1}(\vec{\omega}, \mathbf{r}) \psi_{1}(\vec{\omega}, \mathbf{r})\right\rangle
$$

the anomalous triple correlator

$$
\xi(\vec{\omega}, \mathbf{r}) \equiv\left\langle\psi_{1}^{\dagger}(\vec{\omega}, \mathbf{r}) \psi_{1}(\vec{\omega}, \mathbf{r}) \psi_{1}(\vec{\omega}, \mathbf{r})\right\rangle
$$

and for the operator

$$
\begin{gathered}
\hat{X}(\vec{\omega}, \mathbf{r}) \equiv 2 \psi_{1}^{\dagger}(\vec{\omega}, \mathbf{r}) \psi_{1}(\vec{\omega}, \mathbf{r}) \eta(\vec{\omega}, \mathbf{r})+ \\
+\eta^{*}(\vec{\omega}, \mathbf{r}) \psi_{1}(\vec{\omega}, \mathbf{r}) \psi_{1}(\vec{\omega}, \mathbf{r})+\psi_{1}^{\dagger}(\vec{\omega}, \mathbf{r}) \psi_{1}(\vec{\omega}, \mathbf{r}) \psi_{1}(\vec{\omega}, \mathbf{r}),
\end{gathered}
$$

where, for brevity, the time dependence is not explicitly shown.

Then Eq. (28) yields the equation for the condensate wave function

$$
i \frac{\partial}{\partial t} \eta(\vec{\omega}, \mathbf{r})=w_{1}(\vec{\omega})\left[\frac{(\hat{\mathbf{p}}+m \mathbf{v})^{2}}{2 m}+U(\mathbf{r})-\mu_{0}(\vec{\omega})\right] \eta(\vec{\omega}, \mathbf{r})+
$$




$$
+w_{1}^{2}(\vec{\omega}) \Phi_{0}\left[\rho_{0}(\vec{\omega}, \mathbf{r}) \eta(\vec{\omega}, \mathbf{r})+2 \rho_{1}(\vec{\omega}, \mathbf{r}) \eta(\vec{\omega}, \mathbf{r})+\sigma_{1}(\vec{\omega}, \mathbf{r}) \eta^{*}(\vec{\omega}, \mathbf{r})+\xi(\vec{\omega}, \mathbf{r})\right] .
$$

Equation (29) results in the equation for the field operator of uncondensed atoms

$$
\begin{gathered}
i \frac{\partial}{\partial t} \psi_{1}(\vec{\omega}, \mathbf{r})=w_{1}(\vec{\omega})\left[\frac{\hat{\mathbf{p}}^{2}}{2 m}+U(\mathbf{r})-\mu_{1}(\vec{\omega})\right] \psi_{1}(\vec{\omega}, \mathbf{r})+ \\
+w_{1}^{2}(\vec{\omega}) \Phi_{0}\left[2 \rho_{0}(\vec{\omega}, \mathbf{r}) \psi_{1}(\vec{\omega}, \mathbf{r})+\eta^{2}(\vec{\omega}, \mathbf{r}) \psi_{1}^{\dagger}(\vec{\omega}, \mathbf{r})+\hat{X}(\vec{\omega}, \mathbf{r})\right] .
\end{gathered}
$$

And Eq. (30) gives the equation for the field operator of normal atoms

$$
i \frac{\partial}{\partial t} \psi_{2}(\mathbf{r})=w_{2}\left[\frac{\hat{\mathbf{p}}^{2}}{2 m}+U(\mathbf{r})-\mu_{2}\right] \psi_{2}(\mathbf{r})+w_{2}^{2} \Phi_{0} \psi_{2}^{\dagger}(\mathbf{r}) \psi_{2}(\mathbf{r}) \psi_{2}(\mathbf{r}) .
$$

In these equations, again for brevity, the time dependence is not written explicitly.

When considering a stationary state, it is possible to introduce an effective temperature $T_{\text {eff }}$ characterizing the stationary input of energy into the system [72-74]. Then an averaged behaviour of the latter, in the sense of averaging over the observation time, can be described in terms of effective statistical ensembles and the corresponding thermodynamic quantities $[75,76]$. For stationary classical turbulence, a similar approach has been used by Kraichnan and Montgomery [77].

For the considered case of the stationary quantum turbulence, we can define the grand thermodynamic potential

$$
\Omega=-T_{\text {eff }} \ln \operatorname{Tr} \exp (-\beta \widetilde{H}),
$$

in which $\beta \equiv 1 / T_{\text {eff }}$. The condition of stability for the heterovortex mixture requires that the thermodynamic potential (37) be minimal with respect to the corresponding geometric weights:

$$
\frac{\delta \Omega}{\delta w_{1}(\vec{\omega})}=0, \quad \frac{\delta^{2} \Omega}{\delta w_{1}^{2}(\vec{\omega})}>0
$$

where, to take into account normalization (27), one has to set

$$
w_{2}=1-\int w_{1}(\vec{\omega}) d m(\vec{\omega})
$$

The first of conditions (38) gives

$$
\left\langle\frac{\delta \widetilde{H}}{\delta w_{1}(\vec{\omega})}\right\rangle=0
$$

and the second yields

$$
\left\langle\frac{\delta^{2} \widetilde{H}}{\delta w_{1}^{2}(\vec{\omega})}\right\rangle>\beta\left\langle\left(\frac{\delta \widetilde{H}}{\delta w_{1}(\vec{\omega})}\right)^{2}\right\rangle .
$$

Since the right-hand side of inequality (41) is positive, the necessary stability condition reads as

$$
\left\langle\frac{\delta^{2} \widetilde{H}}{\delta w_{1}^{2}(\vec{\omega})}\right\rangle>0
$$


Let us introduce the notations

$$
\begin{gathered}
K(\vec{\omega}) \equiv \int\left\langle\hat{\psi}(\vec{\omega}, \mathbf{r})\left[\frac{\hat{\mathbf{p}}^{2}}{2 m}+U(\mathbf{r})\right] \hat{\psi}(\vec{\omega}, \mathbf{r})\right\rangle d \mathbf{r} \\
\Phi(\vec{\omega}) \equiv \Phi_{0} \int\left\langle\hat{\psi}^{\dagger}(\vec{\omega}, \mathbf{r}) \hat{\psi}^{\dagger}(\vec{\omega}, \mathbf{r}) \hat{\psi}(\vec{\omega}, \mathbf{r}) \hat{\psi}(\vec{\omega}, \mathbf{r})\right\rangle d \mathbf{r}
\end{gathered}
$$

for the superfluid state with vorticity $\vec{\omega}$ and

$$
\begin{aligned}
K_{2} & \equiv \int\left\langle\psi_{2}^{\dagger}(\mathbf{r})\left[\frac{\hat{\mathbf{p}}^{2}}{2 m}+U(\mathbf{r})\right] \psi_{2}(\mathbf{r})\right\rangle d \mathbf{r}, \\
\Phi_{2} & \equiv \Phi_{0} \int\left\langle\psi_{2}^{\dagger}(\mathbf{r}) \psi_{2}^{\dagger}(\mathbf{r}) \psi_{2}(\mathbf{r}) \psi_{2}(\mathbf{r})\right\rangle d \mathbf{r}
\end{aligned}
$$

for the normal state. And let us define the integrals

$$
R_{0}(\vec{\omega}) \equiv \int \rho_{0}(\vec{\omega}, \mathbf{r}) d \mathbf{r}, \quad R_{1}(\vec{\omega}) \equiv \int \rho_{1}(\vec{\omega}, \mathbf{r}) d \mathbf{r}, \quad R_{2} \equiv \int \rho_{2}(\mathbf{r}) d \mathbf{r}
$$

Then the numbers of condensed and uncondensed atoms in the $\vec{\omega}$ superfluid state, and atoms in the normal state are

$$
N_{0}(\vec{\omega})=w_{1}(\vec{\omega}) R_{0}(\vec{\omega}), \quad N_{1}(\vec{\omega})=w_{1}(\vec{\omega}) R_{1}(\vec{\omega}), \quad N_{2}=w_{2} R_{2},
$$

respectively. Also, we define

$$
\widetilde{K}_{1}(\vec{\omega}) \equiv K(\vec{\omega})-\mu_{0}(\vec{\omega}) R_{0}(\vec{\omega})-\mu_{1}(\vec{\omega}) R_{1}(\vec{\omega}), \quad \widetilde{K}_{2} \equiv K_{2}-\mu_{2} R_{2} .
$$

Employing these notations, from Eq. (40), we find the geometric weight for the superfluid state with vorticity $\vec{\omega}$ in the form

$$
w_{1}(\vec{\omega})=\frac{\Phi_{2}+\widetilde{K}_{2}-\widetilde{K}_{1}(\vec{\omega})}{\Phi(\vec{\omega})+\Phi_{2}} .
$$

¿From the stability condition (42) we obtain

$$
\Phi(\vec{\omega})+\Phi_{2}>0
$$

The system chemical potential can be found from the relations

$$
F=\Omega+\mu N=\Omega+\int\left[\mu_{0}(\vec{\omega}) N_{0}(\vec{\omega})+\mu_{1}(\vec{\omega}) N_{1}(\vec{\omega})\right] d m(\vec{\omega})+\mu_{2} N_{2},
$$

defining the free energy. This yields

$$
\mu=\int\left[\mu_{0}(\vec{\omega}) n_{0}(\vec{\omega})+\mu_{1}(\vec{\omega}) n_{1}(\vec{\omega})\right] d m(\vec{\omega})+\mu_{2} n_{2}
$$

where the corresponding atomic fractions are

$$
n_{0}(\vec{\omega}) \equiv \frac{N_{0}(\vec{\omega})}{N} \quad n_{1}(\vec{\omega}) \equiv \frac{N_{1}(\vec{\omega})}{N}, \quad n_{2} \equiv \frac{N_{2}}{N}
$$


The superfluid and normal phases are in mutual equilibrium, because of which their chemical potentials are connected with each other. The relation between the chemical potentials follows from the condition of equilibrium $\delta F=0$, where the variation is with respect to the numbers of atoms. This is equivalent to the equation

$$
\int\left[\frac{\delta F}{\delta N_{0}(\vec{\omega})} \delta N_{0}(\vec{\omega})+\frac{\delta F}{\delta N_{1}(\vec{\omega})} \delta N_{1}(\vec{\omega})\right] d m(\vec{\omega})+\frac{\delta F}{\delta N_{2}} \delta N_{2}=0 .
$$

Keeping the total number of atoms $N$ fixed implies that

$$
\int\left[\delta N_{0}(\vec{\omega})+\delta N_{1}(\vec{\omega})\right] d m(\vec{\omega})+\delta N_{2}=0
$$

By the meaning of the chemical potentials,

$$
\frac{\delta F}{\delta N_{0}(\vec{\omega})}=\mu_{0}(\vec{\omega}), \quad \frac{\delta F}{\delta N_{1}(\vec{\omega})}=\mu_{1}(\vec{\omega}), \quad \frac{\delta F}{\delta N_{2}}=\mu_{2} .
$$

And for the atomic fractions, we have

$$
\frac{\delta N_{0}(\vec{\omega})}{\delta N}=n_{0}(\vec{\omega}), \quad \frac{\delta N_{1}(\vec{\omega})}{\delta N}=n_{1}(\vec{\omega}), \quad \frac{\delta N_{2}}{\delta N}=n_{2}
$$

With the notation

$$
n_{0} \equiv \int n_{0}(\vec{\omega}) d m(\vec{\omega}), \quad n_{1} \equiv \int n_{1}(\vec{\omega}) d m(\vec{\omega})
$$

normalization (25) becomes

$$
n_{0}+n_{1}+n_{2}=1 .
$$

Invoking the above relations, from the equilibrium condition (53), we obtain

$$
\mu_{2}=\frac{\int\left[\mu_{0}(\vec{\omega}) n_{0}(\vec{\omega})+\mu_{1}(\vec{\omega}) n_{1}(\vec{\omega})\right] d m(\vec{\omega})}{n_{0}+n_{1}}
$$

Substituting this into (51) gives

$$
\mu=\frac{\int\left[\mu_{0}(\vec{\omega}) n_{0}(\vec{\omega})+\mu_{1}(\vec{\omega}) n_{1}(\vec{\omega})\right] d m(\vec{\omega})}{n_{0}+n_{1}}
$$

that is,

$$
\mu=\mu_{2} .
$$

The total superfluid density can be found [33] from the general expression

$$
\rho_{s}=\rho-\frac{\beta}{3 m V} \Delta^{2}(\hat{\mathbf{P}})
$$

in which $\rho=N / V$ is the total atomic density and the momentum dispersion

$$
\Delta^{2}(\hat{\mathbf{P}}) \equiv\left\langle\hat{\mathbf{P}}^{2}\right\rangle-\langle\hat{\mathbf{P}}\rangle^{2}
$$


characterizes the amount of heat dissipated in the system. The total momentum is defined as

$$
\hat{\mathbf{P}} \equiv \lim _{u \rightarrow 0} \frac{\partial \widetilde{H}_{u}}{\partial \mathbf{u}},
$$

where $\widetilde{H}_{u}$ is the Hamiltonian for the system boosted with velocity $u$ (see details in [33]). This gives

$$
\begin{gathered}
\hat{\mathbf{P}}=\hat{\mathbf{P}}_{1}+\hat{\mathbf{P}}_{2}, \quad \hat{\mathbf{P}}_{1}=\int \hat{\mathbf{P}}(\vec{\omega}) d m(\vec{\omega}), \\
\hat{\mathbf{P}}(\vec{\omega})=w_{1}(\vec{\omega}) \int \hat{\psi}(\vec{\omega}, \mathbf{r})(-i \nabla) \hat{\psi}(\vec{\omega}, \mathbf{r}) d \mathbf{r}, \\
\hat{\mathbf{P}}_{2}=w_{2} \int \psi_{2}(\mathbf{r})(-i \nabla) \psi_{2}(\mathbf{r}) d \mathbf{r} .
\end{gathered}
$$

Because of the vanishing covariance

$$
\operatorname{cov}\left(\hat{\mathbf{P}}_{1}, \hat{\mathbf{P}}_{2}\right) \equiv \frac{1}{2}\left\langle\hat{\mathbf{P}}_{1} \hat{\mathbf{P}}_{2}+\hat{\mathbf{P}}_{2} \hat{\mathbf{P}}_{1}\right\rangle-\left\langle\hat{\mathbf{P}}_{1}\right\rangle\left\langle\hat{\mathbf{P}}_{2}\right\rangle=0
$$

we have

$$
\Delta^{2}(\hat{\mathbf{P}})=\Delta^{2}\left(\hat{\mathbf{P}}_{1}\right)+\Delta^{2}\left(\hat{\mathbf{P}}_{2}\right) .
$$

Therefore, the superfluid density (62) takes the form

$$
\rho_{2}=\rho-\frac{\beta}{3 m V}\left[\Delta^{2}\left(\hat{\mathbf{P}}_{1}\right)+\Delta^{2}\left(\hat{\mathbf{P}}_{2}\right)\right] .
$$

Generally, a Bose system, subject to the action of an external alternating field, can be in the following five states, which depend on the modulation amplitude and time, that is, on the amount of energy pumped into the system.

(i) Homogeneous superfluid: If there is no external perturbation, the system is in equilibrium, and the effective temperature equals the usual temperature, $T_{\text {eff }}=T$. We assume that the latter is below the Bose-Einstein condensation temperature, so that the system is Bose condensed and is in a homogeneous superfluid state without vortices. The absence of vortices implies zero vorticity $\vec{\omega}=0$. The fact that the whole system is filled by a homogeneous superfluid, without vortices, means that the geometric weight

$$
w_{1}(0)=1
$$

This state remains under rather weak perturbations, when the effective temperature is yet close to $T<T_{c}$.

(ii) Vortex superfluid: Disturbing the system by an alternating field injects energy into the system. The pumped mechanical energy increases the effective temperature. And, after sufficient amount of energy has been pumped into the system, there appear quantized vortices. First, there arise just a few of them, whose energy approximately equals the pumped energy. This can be expressed through the sum

$$
w_{1}(0)+\sum_{i} w_{1}\left(\vec{\omega}_{i}\right)=1
$$


in which, in addition to the geometric weight $w_{1}(0)$, there appear a few terms $w_{1}(\vec{\omega})$, with $\vec{\omega} \neq 0$, describing a small (roughly speaking, less then ten) number of vortices.

(iii) Turbulent superfluid: When the energy, pumped into the system, reaches a critical value, quantum turbulence develops with a great number of quantized vortices forming a tangle. This can be denoted as

$$
\int w_{1}(\vec{\omega}) d m(\vec{\omega})=1,
$$

the number of vortices being much larger than ten. The integral includes the term $w_{1}(0)$.

(iv) Mixture of turbulent superfluid and normal fluid: A rather strong pumping, not only produces vortices, but starts destroying superfluidity, so that the admixture of the normal (nonsuperfluid) liquid appears. This means that the equation

$$
\int w_{1}(\vec{\omega}) d m(\vec{\omega})+w_{2}=1
$$

becomes valid, with a nonzero geometric weight of the normal fluid $w_{2}>0$.

(v) Normal fluid: When the pumping becomes very strong, it can destroy all BoseEinstein condensate and, hence, superfluid, transferring the system into the normal state. If there exists solely the normal phase, then

$$
w_{2}=1
$$

The normal state can be turbulent, but this would be the classical turbulence.

The overall picture is presented in the scheme of Fig. 1.

\section{Concluding remarks}

A statistical model is suggested, describing stationary states arising in a superfluid subject to the action of an external alternating field pumping energy into the system. The latter is represented as a continuous mixture of dynamic phases with different vorticities. The case of no vortices is included, corresponding to zero vorticity. The possible destruction of superfluidity by the pumping, leading to the developing normal (nonsuperfluid) state is also taken into account. The vortices arise owing to dynamic instability corresponding to the appearance of unstable growing collective excitations.

The first three regimes, shown in the scheme of Fig. 1, that is, homogeneous superfluid, vortex superfluid, and turbulent superfluid, have been realized, by modulating the trapping potential, in experiments $[57,58]$ of the Bagnato group. A detailed analysis of these experiments and their relation to theory will be given in separate publications. Here, we give some estimates for clarifying the physical picture by presenting the typical parameters of a turbulent superfluid.

In experiments $[57,58]$, trapped atoms of ${ }^{87} \mathrm{Rb}$ are cooled down to form Bose-Einstein condensate. Hence, atomic mass is $m=1.445 \times 10^{-22} \mathrm{~g}$ and scattering length is $a_{s}=$ $0.577 \times 10^{-6} \mathrm{~cm}$. Almost all atoms have been condensed, composing the condensate of $N=2 \times 10^{5}$ atoms, thermal fraction being rather small. A cylindrical harmonic trap is 
characterized by the radial frequency $\omega_{\perp}=2 \pi \times 210 \mathrm{~Hz}=1.319 \times 10^{3} \mathrm{~s}^{-1}$ and longitudinal frequency $\omega_{z}=2 \pi \times 23 \mathrm{~Hz}=1.445 \times 10^{2} \mathrm{~s}^{-1}$. The related oscillator lengths,

$$
l_{\perp} \equiv \sqrt{\frac{\hbar}{m \omega_{\perp}}}, \quad l_{z} \equiv \sqrt{\frac{\hbar}{m \omega_{z}}},
$$

are $l_{\perp}=0.744 \times 10^{-4} \mathrm{~cm}$ and $l_{z}=2.248 \times 10^{-4} \mathrm{~cm}$. The average frequency and oscillator length,

$$
\omega_{0} \equiv\left(\omega_{\perp}^{2} \omega_{z}\right)^{1 / 3}, \quad l_{0} \equiv\left(l_{\perp}^{2} l_{z}\right)^{1 / 3}=\sqrt{\frac{\hbar}{m \omega_{0}}},
$$

are $\omega_{0}=0.631 \times 10^{3} \mathrm{~s}^{-1}$ and $l_{0}=1.076 \times 10^{-4} \mathrm{~cm}$. The effective volume of the condensate

$$
V_{\text {eff }} \equiv \pi l_{\perp}^{2} 2 l_{z}=2 \pi l_{0}^{3}
$$

is $V_{\text {eff }}=0.783 \times 10^{-11} \mathrm{~cm}^{3}$. Therefore, the average condensate density is $\rho \equiv N / V_{\text {eff }}=$ $2.554 \times 10^{15} \mathrm{~cm}^{-3}$. The average interatomic distance is $a=\rho^{-1 / 3}=0.732 \times 10^{-5} \mathrm{~cm}$. This shows that $a_{s} \ll a$ and the gas parameters are small: $\rho a_{s}^{3}=0.491 \times 10^{-3}$ and $\rho^{1 / 3} a_{s}=0.079$. That is, the atomic interactions are weak.

The trap is subject to an external field modulation during the time $t_{\text {ext }}=0.02 \mathrm{~s}-0.06$ $\mathrm{s}$ with an alternating potential of frequency $\omega_{\bmod }=2 \pi \times 200 \mathrm{~Hz}=1.257 \times 10^{3} \mathrm{~s}^{-1}$. Thence the modulation period is $t_{\text {mod }} \equiv 2 \pi / \omega_{\text {mod }}=5 \times 10^{-3} \mathrm{~s}$. The local-equilibrium time [29] $t_{l o c}=m / \hbar \rho a_{s}=0.929 \times 10^{-4} \mathrm{~s}$ is much shorter than the modulation period $t_{m o d}$, which implies that the system is always in local equilibrium.

The typical size of a vortex core is the healing length

$$
\xi=\frac{1}{\sqrt{2 m \rho \Phi_{0}}}=\frac{1}{\sqrt{8 \pi \rho a_{s}}},
$$

which is $\xi=0.519 \times 10^{-5} \mathrm{~cm}$, being of order of interatomic distance $a$.

The sound velocity

$$
c=\sqrt{\frac{\rho}{m} \Phi_{0}}=\frac{\hbar}{m} \sqrt{4 \pi \rho a_{s}}
$$

becomes $c=0.994 \mathrm{~cm} / \mathrm{s}$. This defines the coherence length $l_{\text {coh }}=\hbar / m c=0.735 \times 10^{-5} \mathrm{~cm}$ that is of order of the healing length.

The vortex velocity (1), for $n=1$, is of order

$$
v=\frac{\hbar}{2 m l_{0}} \ln \left(\frac{l_{0}}{\xi}\right)
$$

which gives $v=0.103 \mathrm{~cm} / \mathrm{s}$.

Another typical velocity is that characterizing the velocity of atomic collisions $v_{c o l}=$ $\hbar / m a_{s}=12.65 \mathrm{~cm} / \mathrm{s}$ during the collision time $t_{c o l}=a_{s} / v_{c o l}=0.456 \times 10^{-7} \mathrm{~s}$. Thus, the relation between the characteristic velocities is

$$
v \ll c \ll v_{c o l} .
$$

The characteristic vortex energy

$$
\varepsilon_{\text {vor }}=\frac{\hbar}{m l_{0}} \ln \left(\frac{l_{0}}{\xi}\right)
$$


is $\varepsilon=1.912 \times 10^{3} \mathrm{~s}^{-1}$.

Summarizing the relations between the typical times yields

$$
t_{c o l} \ll t_{l o c} \ll t_{\text {mod }} \ll t_{\text {ext }} .
$$

And the relations between the characteristic lengths are

$$
a_{s} \ll \xi \sim a \sim l_{\text {coh }} \ll l_{0} \text {. }
$$

Finally, the turbulent regime has to be formed by many vortices, $N_{v o r}>10$. But there is the maximal number of vortices of order

$$
N_{\max } \sim\left(\frac{l_{0}}{l_{\text {coh }}}\right)^{2} \div\left(\frac{l_{0}}{\xi}\right)^{2}
$$

before the vortices start essentially overlapping with each other. If this overlap becomes too strong, the superfluid state is destroyed. Therefore the number of vortices in a turbulent superfluid is in the interval

$$
10<N_{\text {vor }}<200 \div 400
$$

This is in agreement with experiment [58], where up to 200 vortices were observed.

The turbulent superfluid, being a tangled mixture of many condensates with vortices, should exhibit a specific behaviour, when the trapping potential is switched off. Each particular condensate, being released from the trap, expands anisotropically. But, since the mixture of many condensates, as a whole, is isotropic, all particular anisotropic directions are averaged out, so that the total turbulent cloud expands isotropically, keeping its aspect ratio during the whole free expansion. This effect was observed in experiment [58].

As is mentioned above, a more detailed analysis of these and related experiments will be given in separate publications.

Acknowledgements. I am grateful to V.S. Bagnato for discussions of experiments $[57,58]$. Financial support of the Russian Foundation for Basic Research is appreciated. 


\section{Appendix. Continuous products}

The model of quantum turbulence in superfluids, suggested in this paper, is based on the notion of continuous products. For the self-consistency of the paper, in this Appendix, the main definitions are given, related to continuous products. Such products have been used for treating continuous heterophase mixtures [63,64] and models of continuous random walk $[78,79]$.

Let a complex function $f(x): \mathbb{R} \rightarrow \mathbb{C}$ of a real variable $x \in \mathbb{R}$ be given. Suppose, we consider $x$ in an interval $[a, b]$. Divide this interval on $n$ parts of length $\Delta x_{i}$ so that $\sum_{i=1}^{n} \Delta x_{i}=b-a$. Define the limiting procedure

$$
n \rightarrow \infty, \quad \Delta x_{i} \rightarrow 0 \quad(i=1,2, \ldots, n)
$$

A definite continuous product of the function $f(x)$ on an interval $[a, b]$ is given by the limit

$$
\prod_{x=a}^{b} f(x) \equiv \lim _{n \rightarrow \infty} \prod_{i=1}^{n} f\left(a+\Delta x_{i}\right),
$$

where the above limiting procedure is assumed.

In order to transform this limit to a more tractable form, let us take the logarithm of the limiting equation, which gives

$$
\log \prod_{x=a}^{b} f(x)=\lim _{n \rightarrow \infty} \sum_{i=1}^{n} \log f\left(a+\Delta x_{i}\right) .
$$

The logarithm can be taken over any base. Notice that the right-hand side here is the definition of an integral, so that

$$
\log \prod_{x=a}^{b} f(x)=\int_{a}^{b} \log f(x) d m(x)
$$

where $m(x)$ is a measure on $\mathbb{R}$. Since the logarithm can be taken over any base, we may chose the natural logarithm. Then exponentiation yields another form of the definition for a definite continuous product:

$$
\prod_{x=a}^{b} f(x) \equiv \exp \left\{\int_{a}^{b} \ln f(x) d m(x)\right\} .
$$

The logarithm of a complex function is given by the expression

$$
\ln f(x)=\ln |f(x)|+i \arg f(x) \text {. }
$$

Using the notion of indefinite integrals, it is possible to define an indefinite continuous product

$$
\prod_{x} f(x)=c \exp \left\{\int \ln f(x) d m(x)\right\},
$$


where $c>0$ is a positive constant. Then, we can introduce the action inverse to the continuous product as

$$
f(x)=\exp \left\{\frac{1}{m^{\prime}(x)} \frac{d}{d x} \ln \prod_{x} f(x)\right\},
$$

where $m^{\prime}(x) \equiv d m(x) / d x$.

It is straightforward to generalize the notion of a continuous product as follows. Let a complex function $f(x): \mathbb{D} \rightarrow \mathbb{C}$ be given on a measurable domain $\mathbb{D}$ with a measure $m(x)$. Then the continuous product of $f(x)$ over the domain $\mathbb{D}$ is

$$
\prod_{x \in \mathbb{D}} f(x) \equiv \exp \left\{\int_{x \in \mathbb{D}} \ln f(x) d m(x)\right\} \text {. }
$$

It is possible to introduce continuous products of spaces $[63,64,80]$. Let $y_{x}, z_{x} \in \mathcal{U}_{x}$ be vectors of a unitary space $\mathcal{U}_{x}$ with a scalar product $\left(y_{x}, z_{x}\right)$ and $x \in \mathbb{D}$. The manifold $\mathbb{D}$ is assumed to be measurable, with a measure $m(x)$. The norm of $y_{x}$, generated by the scalar product, is $\left\|y_{x}\right\| \equiv \sqrt{\left(y_{x}, y_{x}\right)}$.

The continuous tensor product of unitary spaces,

$$
\mathcal{U} \equiv \bigotimes_{x \in \mathbb{D}} \mathcal{U}_{x}
$$

consists of the vectors that are continuous tensor products

$$
y \equiv \bigotimes_{x \in \mathbb{D}} y_{x} \in \mathcal{U}
$$

The scalar product in $\mathcal{U}$ is given by

$$
(y, z) \equiv \prod_{x \in \mathbb{D}}\left(y_{x}, z_{x}\right)=\exp \left\{\int_{\mathbb{D}} \ln \left(y_{x}, z_{x}\right) d m(x)\right\} .
$$

Respectively, the norm of $y \in \mathcal{U}$, generated by the scalar product, is

$$
\|y\| \equiv \sqrt{(y, y)}=\exp \left\{\int_{\mathbb{D}} \ln \left\|y_{x}\right\| d m(x)\right\} .
$$

When $\mathcal{U}_{x}$ is complete, it becomes a Hilbert space (complete unitary space). Then $\mathcal{U}$ is also a Hilbert space.

Finally, one can introduce continuous tensor products of operators. Let $\mathcal{H}_{x}$ be a Hilbert space, with $x \in \mathbb{D}$, where $\mathbb{D}$ is measurable with a measure $m(x)$. And let an operator $\hat{A}_{x}$ be given on $\mathcal{H}_{x}$. The operator norm can be defined as

$$
\left\|\hat{A}_{x}\right\| \equiv \sup _{y_{x} \in \mathcal{H}_{x}} \frac{\left\|\hat{A}_{x} y_{x}\right\|}{\left\|y_{x}\right\|} \quad\left(y_{x} \neq 0\right) .
$$

The continuous tensor product of the Hilbert spaces $\mathcal{H}_{x}$ is

$$
\mathcal{H} \equiv \bigotimes_{x \in \mathbb{D}} \mathcal{H}_{x}
$$


similarly to (A.5). An operator $\hat{A}$ on $\mathcal{H}$ is an operator continuous tensor product

$$
\hat{A} \equiv \bigotimes_{x \in \mathbb{D}} \hat{A}_{x}
$$

whose action on $y \in \mathcal{H}$ is given by the tensor product

$$
\hat{A}_{y} y \equiv \bigotimes_{x \in \mathbb{D}} \hat{A}_{x} y_{x} .
$$

The operator norm of $\hat{A} \in \mathcal{H}$, defined in the standard way,

$$
\|\hat{A}\| \equiv \sup _{y \in \mathcal{H}} \frac{\|\hat{A} y\|}{\|y\|} \quad(y \neq 0),
$$

results in the relation

$$
\|\hat{A}\|=\exp \left\{\int_{\mathbb{D}} \ln \left\|\hat{A}_{x}\right\| d m(x)\right\} .
$$

These definitions and properties of continuous products allow us to accomplish all necessary calculations for the considered turbulent superfluid represented as a continuous vortex mixture, in which the role of the index $x$ is played by the vorticity $\vec{\omega}$. 


\section{References}

[1] G.K. Batchelor, The Theory of Homogenous Turbulence (Cambridge University, Cambridge, 1953).

[2] J.O. Henze, Turbulence (McGraw-Hill, New York, 1975).

[3] L.D. Landau and E.M. Lifshitz, Fluid Mechanics ( Butterworth-Heinemann, Oxford, 1987).

[4] Y.L. Klimontovich, Turbulent Motion and Structure of Chaos (Nauka, Moscow, 1990).

[5] U. Frisch, Turbulence (Cambridge University, Cambridge, 1995).

[6] I.M. Khalatnikov, Theory of Superfluidity (Nauka, Moscow, 1971).

[7] S.J. Patterman, Superfluid Hydrodynamics (North-Holland, Amsterdam, 1974).

[8] D.R. Tilley and J. Tilley, Superfluidity and Superconductivity (Adam Higler, Bristol, 1990).

[9] P. Nozières and D. Pines, The Theory of Quantum Liquids: Superfluid Bose Liquids (Addison-Wesley, Redwood, 1990).

[10] R.J. Donnelley, Quantized Vortices in Helium II (Cambridge University, Cambridge, 1991).

[11] C.J. Pethick and H. Smith, Bose-Einstein Condensation in Diliute Gases (Cambridge University, Cambridge, 2008).

[12] W.F. Vinen and J.J. Niemela, J. Low Temp. Phys. 128, 167 (2002).

[13] W.F. Vinen, J. Low Temp. Phys. 145, 7 (2006).

[14] M. Tsubota, J. Phys. Soc. Jap. 77, 111006 (2008).

[15] M.S. Paoletti, M.E. Fisher, K.R. Sreenivasan, and D.P. Lathrop, Phys. Rev. Lett. 101, 154501 (2008).

[16] M. Blazkova, D. Schmoranzer, L. Skrbek, and W.F. Vinen, Phys. Rev. B 79, 054522 (2009).

[17] G.E. Volovik, JETP Lett. 75, 491 (2002).

[18] S.E. Korshunov, JETP Lett. 75, 496 (2002).

[19] K.W. Madison, F. Chevy, V. Bretin, and J. Dalibard, Phys. Rev. Lett. 86, 4443 (2001).

[20] S. Sinha and Y. Castin, Phys. Rev. Lett. 87, 190402 (2001).

[21] N.G. Parker, N.P. Proukakis, C.F. Barenghi, and C.S. Adams, Phys. Rev. Lett. 92, 160403 (2004). 
[22] N.G. Parker and C.S. Adams, Phys. Rev. Lett. 95, 145301 (2005).

[23] K.W. Schwarz, Phys. Rev. B 31, 5782 (1985).

[24] G.P. Bewley, M.S. Paoletti, K.R. Sreenivasan, and D.P. Lathrop, Proc. Nat. Acad. Sci. USA 105, 13707 (2008).

[25] B.V. Svistunov, Phys. Rev. B 52, 3647 (1995).

[26] E.V. Kozik and B.V. Svistunov, e-print arXiv:0904.1379 (2009).

[27] G.P. Bewley, D.P. Lathrop, and K.R. Sreenivasan, Nature 441, 588 (2006).

[28] P.W. Courteille, V.S. Bagnato, and V.I. Yukalov, Laser Phys. 11, 659 (2001).

[29] V.I. Yukalov, Laser Phys. Lett. 1, 435 (2004).

[30] V.I. Yukalov and M.D. Girardeau, Laser Phys. Lett. 2, 375 (2005).

[31] A. Posazhennikova, Rev. Mod. Phys. 78, 1111 (2006).

[32] N.P. Proukakis and B. Jackson, J. Phys. B 41, 203002 (2008).

[33] V.I. Yukalov, Laser Phys. 19, 1 (2009).

[34] N.R. Cooper, Adv. Phys. 57, 539 (2008).

[35] A.L. Fetter, Rev. Mod. Phys. 81, 647 (2009).

[36] S.I. Matveenko, D. Kovrizhin, S. Ouvry, and G.V. Shlyapnikov, Phys. Rev. A 80, 063621 (2009).

[37] R. Rajaraman, Solitons and Instantons (North-Holland, Amsterdam, 1982).

[38] M.J. Ablowitz and P.A. Clarcson, Solitons, Nonlinear Evolution Equations and Inverse Scattering (Cambridge University, Cambridge, 1991).

[39] V.I. Yukalov, E.P. Yukalova, and V.S. Bagnato, Phys. Rev. A 56, 4845 (1997).

[40] V.I. Yukalov, E.P. Yukalova, and V.S. Bagnato, Laser Phys. 10, 26 (2000).

[41] V.I. Yukalov, E.P. Yukalova, and V.S. Bagnato, Laser Phys. 11, 455 (2001).

[42] V.I. Yukalov, E.P. Yukalova, and V.S. Bagnato, Phys. Rev. A 66, 043602 (2002).

[43] V.I. Yukalov, E.P. Yukalova, and V.S. Bagnato, Laser Phys. 13, 861 (2003).

[44] E.R. Ramos, E.A. Henn, J.A. Seman, M.A. Caracanhas, K. Helmerson, V.I. Yukalov, and V.S. Bagnato, Phys. Rev. A 78, 063412 (2008).

[45] V.I. Yukalov and V.S. Bagnato, Laser Phys. Lett. 6, 399 (2009).

[46] V.I. Yukalov, K.P. Marzlin, and E.P. Yukalova, Laser Phys. 14, 565 (2004). 
[47] V.I. Yukalov, K.P. Marzlin, and E.P. Yukalova, Phys. Rev. A 69, 023620 (2004).

[48] S.K. Adhikari, Phys. Lett. A 313, 211 (2003).

[49] M. Kobayashi and M. Tsubota, Phys. Rev. A 76, 045603 (2007).

[50] D. Proment, S. Nazarenko, and M. Onorato, Phys. Rev. A 80, 051603 (2009).

[51] V.I. Yukalov, Laser Phys. Lett. 3, 406 (2006).

[52] D. Ruelle, Physica D 7, 40 (1983).

[53] E.A. Kuznetsov, A.M. Rubenchik, and V.E. Zakharov, Phys. Rep. 142, 103 (1986).

[54] K. Kaneko, Physica D 34, 1 (1989).

[55] S.N. Gurbatov, A.N. Malakhov, and A.I. Saichev, Nonlinear Random Waves in Nondispersive Media (Nauka, Moscow, 1990).

[56] H. Fujisaka, K. Egami, and T. Yamada, Phys. Lett. A 174, 103 (1993).

[57] E.A. Henn, J.A. Seman, E.R. Ramos, M. Caracanhas, P. Castilho, E.P. Olimpio, G. Roati, D.V. Magalhães, K.M. Magalhães, and V.S. Bagnato, Phys. Rev. A 79, 043618 (2009).

[58] E.A. Henn, J.A. Seman, G. Roati, K.M. Magalhães, and V.S. Bagnato, Phys. Rev. Lett. 103, 045301 (2009).

[59] V.I. Yukalov, Theor. Math. Phys. 26, 274 (1976).

[60] V.I. Yukalov, Theor. Math. Phys. 28, 652 (1976).

[61] V.I. Yukalov, Phys. Lett. A 81, 249 (1981).

[62] V.I. Yukalov, Phys. Lett. A 81, 433 (1981).

[63] V.I. Yukalov, Physica A 108, 402 (1981).

[64] V.I. Yukalov, Phys. Rep. 208, 395 (1991).

[65] V.I. Yukalov, Int. J. Mod. Phys. B 17, 2333 (2003).

[66] V.I. Yukalov, Symmetry 2, 40 (2010).

[67] V.I. Yukalov, Phys. Rev. E 72, 066119 (2005).

[68] V.I. Yukalov, Phys. Lett. A 359, 712 (2006).

[69] V.I. Yukalov, Ann. Phys. 323, 461 (2008).

[70] V.I. Yukalov, Laser Phys. Lett. 4, 632 (2007).

[71] N.N. Bogolubov, Lectures on Quantum Statistics (Gordon and Breach, New York, 1970). 
[72] R.H. Fowler and E.A. Guggenheim, Statistical Thermodynamics (Cambridge University, Cambridge, 1939).

[73] M.A. Leontovich, Introduction to Thermodynamics (Nauka, Moscow, 1983).

[74] R.L. Stratonovich, Nonlinear Nonequilibrium Thermodynamics (Springer, Berlin, 1994).

[75] A.Y. Khinchin, Mathematical Foundations of Statistical Mechanics (Dover, New York, 1960).

[76] A. Isihara, Statistical Physics (Academic, New York, 1971).

[77] R. Kraichnan and D. Montgomery, Rep. Prog. Phys. 43, 547 (1980).

[78] S.I. Frolov, J. Math. Sci. 88, 884 (1998).

[79] B. Tsirelson, Prob. Surv. 1, 173 (2004).

[80] A. Guichardet, Symmetric Hilbert Spaces and Related Topics (Springer, Berlin, 1972). 


\begin{tabular}{c|c} 
normal fluid & $T_{\text {eff }} \quad w_{2}=1$ \\
\hline $\begin{array}{c}\text { turbulent superfluid } \\
\text { and normal fluid }\end{array}$ & $\int w_{1}(\vec{\omega}) d m(\vec{\omega})+w_{2}=1$ \\
\hline turbulent superfluid & $\int w_{1}(\vec{\omega}) d m(\vec{\omega})=1$ \\
\hline vortex superfluid & $w_{1}(0)+\sum_{i} w\left(\vec{\omega}_{i}\right)=1$ \\
\hline $\begin{array}{c}\text { homogeneous } \\
\text { superfluid }\end{array}$ & $w_{1}(0)=1$ \\
\hline
\end{tabular}

Figure 1: Scheme of the sequence of states for a superfluid subject to the action of an alternating external field. 